\title{
Structure Design and Calculation Analysis on Lateral Current Collector of Hanging Air Train
}

\section{Li-Chao WANG ${ }^{1}$, Xiao-Jun LV ${ }^{1}$, Fang WANG ${ }^{2}$, Chun-Shan YAO $^{2, \text { a }}$ and Chun-Yan $\mathrm{DI}{ }^{2}$}

${ }^{1}$ CRRC Qinqdao SIFang Co., Ltd., Technology Center, Qingdao, China

${ }^{2}$ Changchun Wonderlong Railway Vehicle Equipment Co. Ltd. China

a13341572112@163.com, byxzhu@jlu.edu.cn

Keywords: Lateral Current Collecting Device (CCD), Translational mechanism, Shoe, Spring, Contact force.

\begin{abstract}
The development of this lateral CCD is based on the technical specifications for the hanging air trains. This document describes the main technical parameters of the lateral CCD, structure design , key points, and attentions etc., makes the design calculations on the dynamic part---spring, thus deducing the relations between the contact force of shoe against power rail and the main technical parameters of the mechanism. This CCD adopts a four-rod link translational mechanism in which a new design on the follower lever is introduced to improve the manufacturability of the four-rod mechanism and realize much higher reliability and stability as well as enhanced adaptability of the shoe to the power rail. Both the trial-manufacture of and the tests about this lateral CCD prototype can come up to the technical requirements for the lateral CCD. This later CCD is of compact structure, light weight, good quality and reliability.
\end{abstract}

\section{Introduction about Current Collecting Device}

There are two common ways for the power supply of the rail vehicles, one way is collecting current from the overhead catenary through pantograph; another way is collecting current from the power rail through CCD. Compared to the overhead catenary, the steel-aluminum composite contact rail (the power rail or the third rail) supply system features long service life, stable operation, few maintenance and easy management etc., thus attracting more and more design institutes and users [1]. The CCD manufactures abroad mainly are: Stemmann from Germany, Schunk from Austria, Faiveley from France, Wabtec from USA, and Brecknell willis from Britain [2]. The power rail is the third rail for power supply aside from the two running rails of the train. The current collecting device --- current collector takes power from the third rail while contacting with and sliding on it and then transmits the power to the train.

In worldwide, according to the contact mode of the CCD shoe against the power rail, the CCDs are usually divided into: top contact $\mathrm{CCD}$, bottom contact $\mathrm{CCD}$, lateral contact $\mathrm{CCD}$ and dual lateral contact CCD. Currently in China, the contact modes of CCD shoes against third rails are mostly top contact and bottom contact, which are referred as upper mode and lower mode respectively. Tianjin Metro Line 1, Beijing Metro Line 5, Beijing Metro Line 2, Beijing Metro Line 10 Phase I, Beijing Metro Line 9, Beijing Metro Yizhuang Line, Beijing Metro Line 15, Beijing Metro Fangshan Line, Beijing Metro Changping Line all adopt upper mode while Wuhan Light Rail Phase I, Guangzhou Metro Line 4, Guangzhou Metro Line 5, Tianjin Metro Line 2, Shenzhen Line 3, Wuhan Line 2 and Beijing Yanfang Line all use lower mode. Currently, the DC750V and DC1500V third rail supply systems are commonly used. According to the dynamic source, CCDs can be divided into three types: Mechanical type, Pneumatic type, Mechanical and Pneumatic Hybrid type.

Changchun Wonderlong Rail Vehicle equipment Co., Ltd., together with CRRC Sifang Co., Ltd., 
developed a mechanical lateral CCD which will be used on the Hanging air trains.

\section{Main Technical Parameters for the Lateral CCD}

In accordance with the technical requirements for the CCDs used on the hanging trains, this lateral CCD adopts mechanical structure, uses spring as a kind of dynamic source part, has the shoe contacted with the third rail from the lateral side, i.e, lateral contact, and connects to bogie through insulating base. Conveying the current from the power rail to the train requires the mechanism to be simple, compact and light weighted. The specific parameters are as table1:

\section{Structure Design of the Lateral CCD}

Based on the systematic study on the running posture of the hanging train, power rail layout method and characters, technical requirements for dynamic current collection, electrical insulation requirements, and dynamic friction pairs matching requirements, we scientifically and rationally hammer out the shoe materials and working range, the contact force of shoe against power rail, insulating protection for the lateral CCD etc. This lateral CCD comes up to the working demands of dynamic current collection, reduces the maintenance needs for the $\mathrm{CCD}$, realizes the dynamic stability and current collection reliability and provides power supply security for the stable operation of hanging trains.

Table 1. Technical Parameters for Lateral CCD

\begin{tabular}{|c|c|}
\hline Rated Working Voltage & DC750V(as per GB/T 10411-2005) \\
\hline Working Voltage Range & DC500V DC900V \\
\hline Static Rated Current & $200 \mathrm{~A}$ \\
\hline Dynamic Rated Current & $600 \mathrm{~A}$ \\
\hline Designed Running Speed & $55 \mathrm{~km} / \mathrm{h}$ \\
\hline Static Contact Force & $120 \pm 24 \mathrm{~N}$ \\
\hline Height of CCD at Retraction Position & $\leq 143 \mathrm{~mm}$ \\
\hline Min. Working Height & $150 \mathrm{~mm}$ \\
\hline Nominal Working Height & $185 \mathrm{~mm}$ \\
\hline Max. Working Height & $229 \mathrm{~mm}$ \\
\hline Max. Stretching scope & $230 \mathrm{~mm}$ \\
\hline Electric Clearance & $\geq 30 \mathrm{~mm}$ \\
\hline Creepage Distance & $\geq 35 \mathrm{~mm}$ \\
\hline Total Mass & $\leq 20 \mathrm{Kg}$ \\
\hline
\end{tabular}

The shoe of this lateral CCD keeps smooth contact with the power rail, maintains a certain contact force within $120 \mathrm{~N} \pm 24 \mathrm{~N}$. The smooth and steady movement of the shoe is supported by a four- bar-link translational mechanism which uses self-lubricating sliding bearings for the smooth movement of the revolute pair. The length of the four-bar-link mechanism is determined by the mounting space and working limits of the lateral CCD. The four-bar-link mechanism includes holder, active rod, follower rod, shoe assembly, and the bolts connecting the holder and insulating plate. The shoe assembly consists of shoe, protective base, conductive copper plate, and shoe support etc. The shoe, which can be made of carbon copper alloy or cast iron, has good electrical conductivity, wear resistance and impact resistance. The conductive copper plate is connected with the output cable to transfer the current collected from the power rail by the shoe. The protective base plays a role of damping to avoid overlarge impact on the shoe. The shoe support is driven by the active rod and guarantees a certain 
moving posture. The driving force mechanism uses torsion springs as a force source. The thrust force generated from the torsion springs enables the four-rod-link mechanism revolving around the pin shaft to let the shoe reach the required position.

The shoe of lateral CCD for hanging air trains contacts with the lateral side of power rail, the power rail is installed with its friction surface vertically standing, thus it is difficult for dust, rain, snow and other debris to accumulate on the friction surface of the power rail, and the wear caused by the debris and arc erosion on the power rail and shoe would be reduced. By left and right fishplates, the CCD can smoothly and steadily passes through the turnout and realizes the purpose of steadily collecting current ${ }^{[3]}$. The three dimensional structure of the CCD is shown as Fig 1.

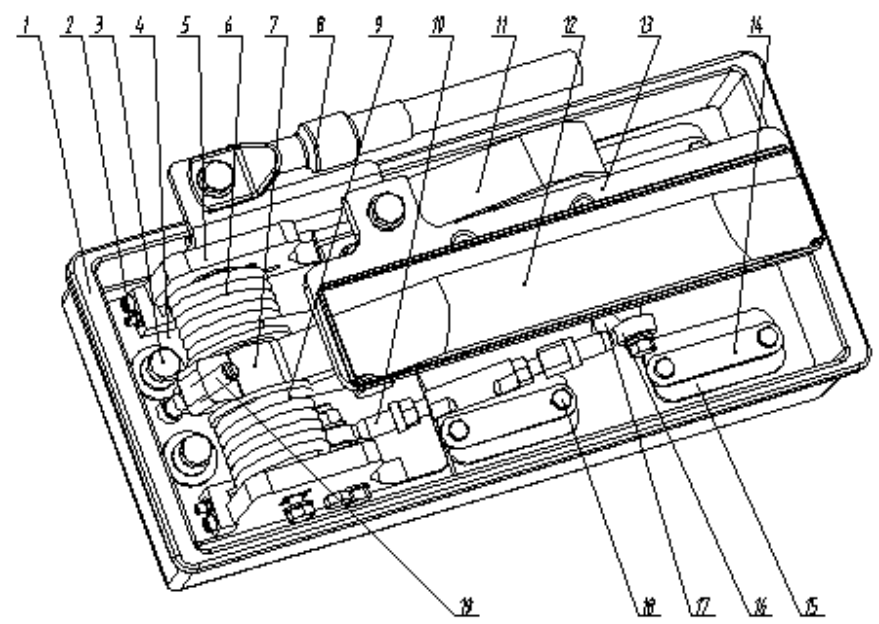

1-insulating base: 2 - force adjustment screw: 3 - bolt connecting the holder and insulating base: 4 -insulating plate: 5-holder : 6-torsion spring: 7—active rod: 8-cable: 9-cardinal axis of torsion spring: 10 - follower rod assembly: 11—output cable: 12 - shoe: 13 - connect copper: 14—stopping pad: 15-small insulating cover: 16-ball joint: 17-lower limit stopping pad: 18 - fitting screw: 19- upper limit stopping pad

Fig. 1. 3D diagram of the lateral CCD

The insulating base of the CCD is connected to bogie through four M12 bolts which are protected by insulating cap. The insulating plate fixed in the insulating base provides insulation security between CCD and bogie.

\section{Analysis on the Four-rod-link Mechanism}

A four- bar-link Translational Mechanism is used to support the shoe movement, self-lubricating sliding bearings are installed inside the revolute pair for the smooth movement. The length of the four-bar-link mechanism is determined by the mounting space and working limits of the lateral CCD. The four-bar-link mechanism includes holder, active rod, follower rod, and shoe assembly etc. The length of each rod of the four-rod-links is worked out by the Sketch Module ${ }^{[4]}$ in the software CATIA according to the mounting space of the CCD. At the free position, the lateral CCD is within a space of $404 \mathrm{mmX} 184 \mathrm{mmX} 230 \mathrm{~mm}$. In Sketch Module, the active rod can be rotated under the condition of the dimension and position under-constraint and marking dimensions can be done under the condition of over-constraint, thus we can work out the shoe's lower limit $150 \mathrm{~mm}$, nominal working position $185 \mathrm{~mm}$, upper limit $229 \mathrm{~mm}$ and the rotating angel of active rod and the corresponding swiping area limit when the shoe is at the $230 \mathrm{~mm}$ free position. When the retracted position is considered as the Zero position, then the relative positions are $15 \mathrm{~mm}, 50 \mathrm{~mm}, 94 \mathrm{~mm}, 95 \mathrm{~mm}$. Taking the $185 \mathrm{~mm}$ nominal working position as an example, the rotating angel of the active rod is 17.105 degree shown as Fig. 2. By Graphic analytic calculation, the rotating angels of the rod corresponding to the four positions are 
respectively 5.062, 17.105, 33.569 and 33.974 .

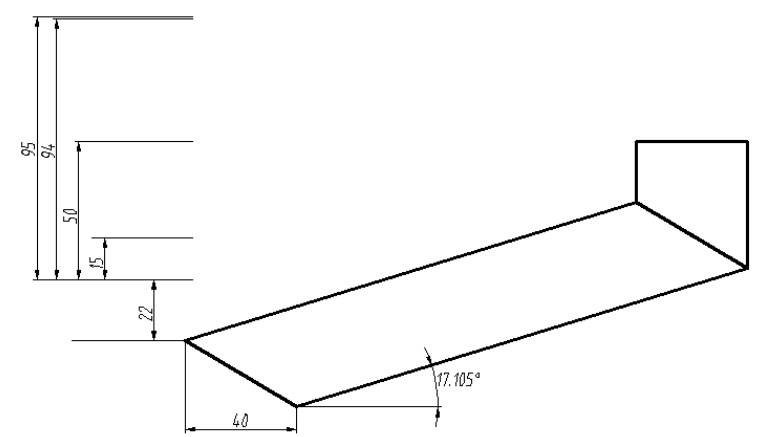

Fig. 2. Rotating Angle of the Active Rod When the Shoe is at $185 \mathrm{~mm}$ Working Position

The four-rod link translational mechanism is in a parallelogram shape, so requires two opposite sides in the same length and parallel. The dimensional errors happened in processing and manufacturing and the shape and position errors could affect the translational motion of the shoe. To obtain good translational motion effect, higher processing and manufacturing precision is required and the cost would be increased accordingly. We designed a combined follower-rod mechanism, thus reduced the requirement for the processing and manufacturing precision, realized higher reliability and stability, and enhanced the adaptability of the shoe to the power rail. The length of the follower-rod can be adjusted through the screw joint, and the depth of parallelism between the active rod and follower rod can be adjusted by the ball joint. The follower rod assembly is shown as Fig. 3.

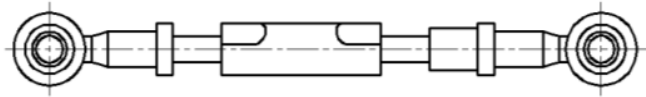

Fig. 3. Follower Rod Assembly

\section{Torsion Spring Design for the Lateral CCD}

The torsion springs provide the shoe with pressure. The contact force between the shoe and the third rail is $120 \pm 24 \mathrm{~N}$. The torsion springs are made of $1 \mathrm{Cr} 18 \mathrm{Ni} 9$ stainless steel wire and the elasticity modulus is $193 \mathrm{GPa}$. The force diagram for the lateral CCD is shown as Fig.4.

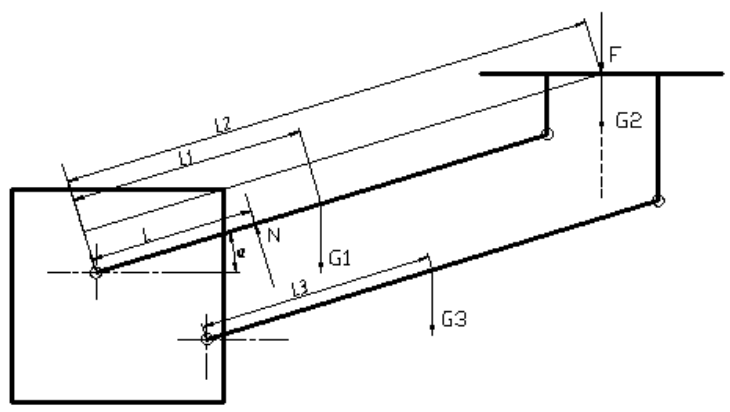

Fig. 4. Force Diagram of the Mechanism

G1: Weight of active rod; G2: Weight of follower rod; G3: Weight of the shoe assembly; $\alpha$ : Angel between the active rod and horizontal line; N: Elastic force generated by the torsion spring; L: Force arm of the elastic force; F: Pressure between the third rail and shoe; L1, L2, L3: the acting positions of 
G1, G2, G3 and F.

The following formula is for any working position of the shoe

$\mathrm{NXL}=\mathrm{G} 1 \mathrm{X} \cos \alpha \mathrm{XL} 1+(\mathrm{G} 2+\mathrm{F}) \mathrm{X} \cos \alpha \mathrm{XL} 2+\mathrm{G} 3 \cos \alpha \mathrm{XL} 3$

$$
F=\frac{N X L-(G 1 X L 1+G 2 X L 2+G 3 X L 3) X \cos \alpha}{L 2 X \cos \alpha}
$$

Torsion angles of the torsion spring corresponding to each working position of the shoe of lateral CCD are shown as Fig. 5.

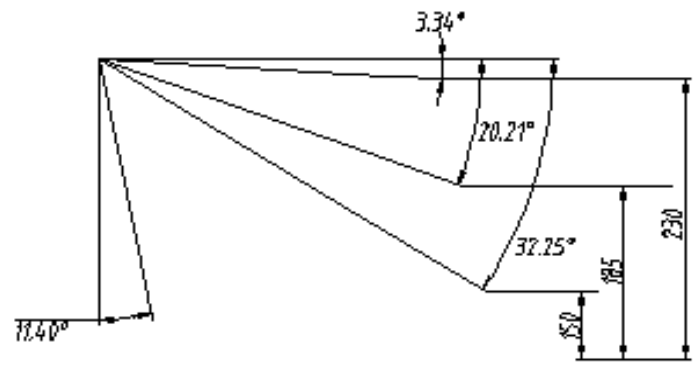

Fig. 5. Torsion Angles of the Torsion Spring

Stiffness of the torsion spring ${ }^{[5]}$ :

$$
T^{\prime}=\frac{E d^{4}}{3667 D n}
$$

E: Elasticity modulus of the torsion spring

$\mathrm{d}$ : Diameter of the spring wire

D: Intermediate diameter of the torsion spring

$\mathrm{n}$ : Number of effective turns of the torsion spring

$T^{\prime}=\frac{E d^{4}}{3667 D n}=\frac{193 \times 10^{9} \times\left(6 \times 10^{-3}\right)^{4}}{3667 \times 48 \times 10^{-3} \times 6}=237 \mathrm{Nmm} /\left(^{\circ}\right)$

When the shoe is at free position, the angel of the torsion spring is 130 , different shoe position height corresponds to different angels of the torsion spring:

When the shoe is at the height of $150 \mathrm{~mm}$, the torsion angel of the torsion spring is $130-(90-11.4-32.25)=83.75^{\circ}$;

When the shoe is at the height of $185 \mathrm{~mm}$, the torsion angel of the torsion spring is $130-(90-11.4-20.21)=71.61^{\circ}$;

When the shoe is at the height of $230 \mathrm{~mm}$, the torsion angel of the torsion spring is $130-(90-11.4-3.34)=54.74^{\circ}$;

And the corresponding torques of the torsion spring are:

When the shoe is at the height of $150 \mathrm{~mm}$, the torque of the torsion spring is $237 X 83.75=19848.75$ N.mm;

When the shoe is at the height of $185 \mathrm{~mm}$, the torque of the torsion spring is 237X71.61=16971.57N.mm;

When the shoe is at the height of $230 \mathrm{~mm}$, the torque of the torsion spring is 237X54.74=12973.38N.mm.

The mechanical structure adopts two torsion springs, according to the Fig.4 and the formula about the shoe pressure, the forces of the shoe at different positions are:

$$
\mathbf{F}=\frac{2 \mathrm{XNXL}-(\mathbf{G} 1 \mathrm{XL} 1+\mathbf{G} 2 \mathrm{XL} 2+\mathbf{G} 3 \mathrm{XL} 3) \mathrm{X} \cos \alpha}{\mathrm{L} 2 \mathrm{X} \cos \alpha}
$$

When the shoe is at the position of $150 \mathrm{~mm}$ 


$$
\begin{aligned}
& \mathbf{F}=\frac{2 \mathrm{X} 19848.75-(8.4672 \mathrm{X} 85+46.06 \mathrm{X} 210.5+5.88 \mathrm{X} 85) \mathrm{X} \cos 5.062^{\circ}}{210.5 \mathrm{X} \cos 5.062^{\circ}}=137.5 \mathrm{~N} \\
& \text { When the shoe is at the position of } 185 \mathrm{~mm} \\
& \mathbf{F}=\frac{2 \mathrm{X} 16971.57-(8.4672 \mathrm{X} 85+46.06 \mathrm{X} 210.5+5.88 \mathrm{X} 85) \mathrm{X} \cos 17.105^{\circ}}{210.5 \mathrm{X} \cos 17.105^{\circ}}=117.15 \mathrm{~N} \\
& \text { When the shoe is at the position of } 230 \mathrm{~mm} \\
& \mathbf{F}=\frac{2 \mathrm{X} 12973.38-(8.4672 \mathrm{X} 85+46.06 \mathrm{X} 210.5+5.88 \mathrm{X} 85) \mathrm{X} \cos 33.974^{\circ}}{210.5 \mathrm{X} \cos 33.974^{\circ}}=96.8 \mathrm{~N}
\end{aligned}
$$

The pressure of the lateral CCD shoe against the third rail is within $120 \pm 24 \mathrm{~N}$, the calculation shows that this contact force between the shoe and third rail meets the technical requirements. The lateral CCD prototype accepted a test of force on its shoe by the force transducer, shown as Fig.6.

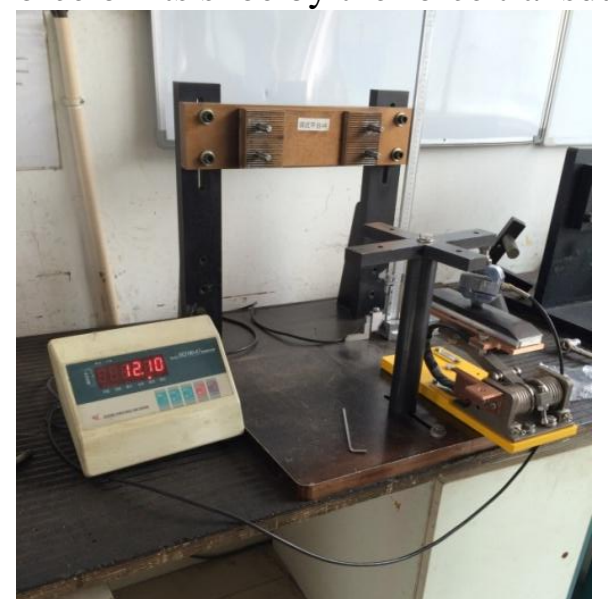

Fig. 6. Force Test on Lateral CCD

\section{Conclusion}

After calculation and routine test, the independently developed CCD for hanging trains meets the requirements of technical specification, features good performance and compact structure. A new design is introduced on the follower rod of the four-rod-link mechanism, which improved the manufacturability of the four-rod-link mechanism, reduced the requirement for manufacturing precision and cost, enhanced the adaptability of the shoe to the power rail, and has a referential meaning to the spring design. The completion of this design of CCD for hanging trains successfully provides a strong guarantee for the hanging trains, has a significant meaning for the busy city traffic to meet the standard of low carbon, green travel, made a try on the CCD localization and simplification.

\section{References}

[1] Wang Zhenquan, Li Xiangquan. Analysis on the Structure and Performance of Split-type Current Collector. Railway Standard Design, 1, 125-127(2011)

[2] Zhang Haifeng, Chen Hengqian, Guo Yanmei. Research of the Third Rail Current Collector. Electric Drive for Locomotives, 6, 46-47(2013)

[3] Li Ning, Chen Ge. Current collection modes choice and collecto structure design of the mid-/low-speed EMS maglev train. Electric Locomotives \& Mass Transit Vehicles, 3, 14-19(2007)

[4] Pan Zhigang, Zhu Yuxiang, Pan Zijian. CATIA V5R21 Practical Basic Tutorial.Beijing Science and Technology Publishing Co., Ltd., 26-42(2015)

[5] Cheng Daxian, Wang Defu, Ji Kuisheng. Mechanical Design Handbook. Fifth edition. Beijing Chemical Industry Publishing Co., Ltd., 11-44-11-46(2008) 